Proceedings of the 2011 Winter Simulation Conference

S. Jain, R. R. Creasey, J. Himmelspach, K. P. White, and M. Fu, eds.

\title{
ESTIMATION AND MANAGEMENT OF PANDEMIC INFLUENZA TRANSMISSION RISK AT MASS IMMUNIZATION CLINICS
}

\author{
Michael F. Beeler \\ Dionne M. Aleman \\ Michael W. Carter \\ Department of Mechanical and Industrial Engineering \\ University of Toronto \\ 5 King's College Road \\ Toronto, M5S 3G8, CANADA
}

\begin{abstract}
Mass immunization clinics (MICs) have become an essential component of pandemic influenza response strategies. By deploying large volumes of vaccines at centralized locations, public health authorities can reduce the complexity of emergency vaccine distribution while also enabling rapid, large-scale vaccination. The risk of influenza transmission at MICs must be understood and mitigated to maximize their effectiveness. We have developed a discrete-event simulation of an MIC that can estimate the expected number of infections resulting from disease transmission within the facility. A simulation experiment is conducted that varies MIC crowdedness, staffing levels and the percentage of infectious individuals entering the MIC-symptomatic or not-to assess the impact of these factors on expected infections. It is shown that the number of expected infections occurring in the MIC, though a small fraction of the influenza cases likely averted due to vaccination, is large enough to warrant mitigation measures.
\end{abstract}

\section{INTRODUCTION}

Mass immunization clinics (MICs) have the potential to help large proportions of the population protect themselves against pandemic influenza by allowing quick, large-scale deployment of vaccines without overwhelming hospitals, existing walk-in clinics and physician offices. In 2001, the Canadian government entered a 10-year pandemic influenza vaccine pre-purchase agreement with domestic manufacturers under the belief that enough vaccine should be available to cover the entire population within a matter of months in the event of a severe pandemic (H. Branswell 2009). The rate of vaccine deployment required to achieve such high and rapid coverage puts significant demands on MICs and the staff who run them. When surges in cases occur, demand for vaccination may swell, leaving many MICs with a surplus of patients, congestion, and long queues. Understanding influenza transmission risk in MIC queues and waiting areas is essential to identifying how to manage those risks to ensure MIC effectiveness.

This simulation study outlines a method for estimating the expected value of new influenza infections occurring at an MIC using a discrete event simulation that models an MIC's daily operations. A designed experiment with three factors is used to assess the impact of (1) changing spacing between MIC clients, (2) changing staffing levels, and (3) increasing the percentage of infectious individuals on the expected number of infections. The MIC model is designed to resemble MICs from the City of Toronto, Ontario, Canada during the $2009 \mathrm{H} 1 \mathrm{~N} 1$ pandemic. This paper outlines the conceptual framework, implementation, and results of the simulation experiment, but is not intended to predict or replicate future or historical MIC performances at specific facilities. The model parameters pertaining to service times, patient arrival rates, and queue length maximums can be adjusted to reflect the realities of different facilities, regions, and pandemics. 


\section{Beeler, Aleman, and Carter}

Simulation modeling has been used extensively to understand the potential dynamics of influenza spreading at the international (Khan et al. 2011), regional (Stroud et al. 2007, Stroud 2006), and municipal levels (Aleman et al. 2010, Das et al. 2008). However, no studies have been conducted on transmission risks within MICs. Most studies of infection risk at the level of particular venues focus specifically on using analytical models to estimate hospital-acquired infection risks (Noakes and Sleigh 2009, Barnes et al. 2010), though a few studies on influenza spread within university campuses have also been conducted (Araz et al. 2011, Mei et al. 2010). This study is the first to use discrete-event simulation to estimate pandemic influenza transmission risks at an individual level in a public venue.

\section{MODEL OVERVIEW}

The flow of patients through an MIC involves a modest number of decision points, queues and processes, as illustrated in Figure 1.

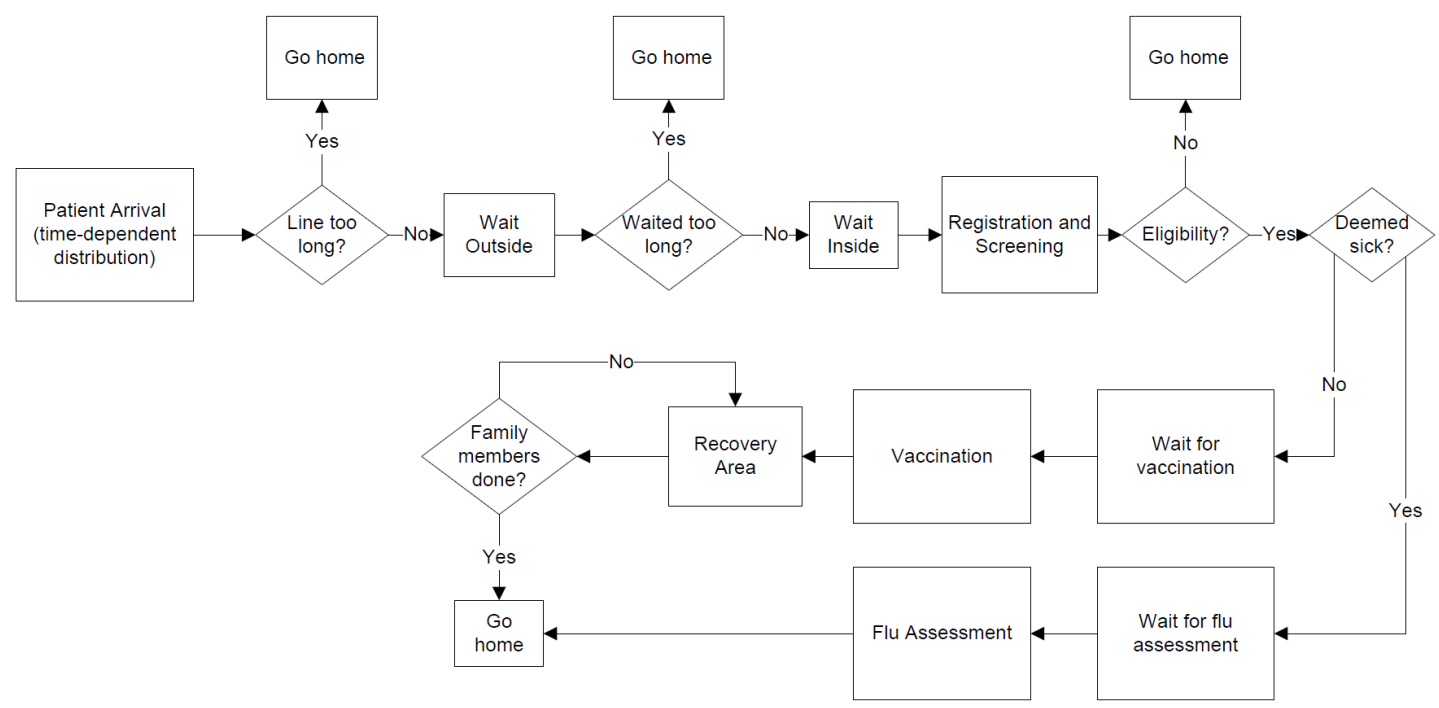

Figure 1: Flowchart of patient movement through MIC.

\subsection{Patient Arrival}

Patients arrive at the MIC facility and must wait outside if the facility is closed or too crowded. Patients are assumed to arrive in family/friend batches of sizes ranging from 1-4, starting 30 minutes before official facility opening time at 8:30 a.m. The inter-arrival time between batches was set to a time-dependent exponential distribution with a rate of 1.33 arrivals per minute from 8:00 a.m. to 4:00 p.m., but linearly diminishing to zero between 4:00 p.m. and facility closure at 6:00 p.m. The simulation assigns each patient one of the following health status labels at the time of their arrival in the system: susceptible, infectiousasymptomatic, infectious-symptomatic, or non-infectious-symptomatic. Patients are also assigned an age label since the transmission probabilities used in the model are age-dependent (Haber et al. 2007).

The patients are also assigned labels indicating whether they belong to a government-defined priority group and whether they have eligible insurance. The distribution of family batch sizes, health label frequencies, priority group membership, and patient arrival rates are set to a user-defined probability profile that can be adapted to the specific policy and pandemic scenarios and demographic assumptions the investigator wishes to test. A list of parameters used in the baseline version of this model is presented in 


\section{Beeler, Aleman, and Carter}

Table 1 . These parameters are meant to simulate a medium-severity pandemic in which a sizable proportion of the population is infectious.

Table 1: Summary of patient parameters.

\begin{tabular}{l|ll}
\hline Parameter & Value & Notes \\
\hline Patient batch arrival rate & $1.33 /$ minute & Tapers off from 4:00 p.m. - 6:00 p.m. \\
$\operatorname{Pr}($ batch size $=1,2,3,4)$ & $0.60,0.25,0.10,0.05$ & Provisional estimates \\
$\operatorname{Pr}($ susceptible $)$ & 0.94 & assumes moderate-severe pandemic \\
$\operatorname{Pr}($ non-infected-symptomatic) & 0.02 & symptoms due to other causes \\
$\operatorname{Pr}($ contagious-symptomatic) & 0.02 & varies with severity of pandemic \\
$\operatorname{Pr}($ contagious-asymptomatic) & 0.02 & may vary according to virus properties \\
$\%$ Age $\in(0-4,5-18,19-64, \geq 64)$ & $5.84,18.06,63.38,12.72$ & (Statistics Canada 2006) \\
\hline
\end{tabular}

\subsection{Service within the MIC}

Once inside the MIC, patients line-up to register for immunization. If the registration queue's capacity is exceeded, patients will be forced to wait outside the facility. The queues for all work stations within the MIC were assigned maximum capacities; if a patient wished to move to a new work station that had no available queue space, the patient would continue to occupy space at the original work station until space in the queue for the next station became available. At registration, patients may be screened so that symptomatic patients are directed to go to the on-site flu assessment center where they will consult with a nurse before going home, or otherwise to leave the facility directly. The effectiveness of screening can be set to less than $100 \%$ to reflect the possibility that some patients with mild flu-like symptoms may want vaccination and consequently claim to be feeling fine to avoid being sent away.

At registration, patients may also be assessed for eligibility depending on whether government policy mandates that vaccines be issued to specific priority groups based on age (e.g., seniors and children only), employment status (e.g., healthcare workers), or pre-existing medical conditions (e.g., immunocompromised persons). Patients who do not have influenza may appear to be sick and may also be sent to the flu assessment center. Patients who have a susceptible label or a contagious-asymptomatic label will be deemed eligible for vaccination unless a specific policy restricting vaccination to priority groups is in place. These patients will enter a new waiting area and stand in one of several queues for a vaccination station. After being vaccinated, patients wait in a recovery area for 10 minutes in case they have an adverse reaction to the vaccine. After the 10 minute period, the patient proceeds to leave the facility.

Each point of service - registration, flu assessment and vaccination-is assigned a distribution representing variation in service times per patient, as well as a certain number of staff, each of whom serves one patient at a time. In the simulation software, points of service are represented as work centers, and staff are represented as replications of the service center. The queue for vaccine administration is given a maximum capacity reflecting the space constraints of the MIC facility. The parameters used for each of these work centers in the baseline model are summarized in Table 2. The service time distributions display the mean and standard deviation in that order. A lognormal distribution was used for registration time to allow for significant variation relative to the absolute size of the mean, while preventing negative service time values.

Table 2: Work center service times and replications.

\begin{tabular}{llll}
\hline Work Center & Service Time & Replications & Queue Capacity \\
\hline Registration & Lognormal $(1.5,0.5)$ & 4 & 20 \\
Flu Assessment & Normal $(10,2.5)$ & 1 & 20 \\
Vaccination & Normal $(6,1)$ & 10 & 90 \\
\hline
\end{tabular}




\section{Beeler, Aleman, and Carter}

\subsection{Modeling Patient Patience, Line Tolerance and Closure}

The simulation also assigns each patient a patience level that determines how long they are willing to wait to merely get in the door of the facility before losing patience and leaving. In the baseline scenario, patience levels were assumed to have a normal distribution of 90 minutes with a standard deviation of 20 minutes. The simulation also assigns a line length tolerance value to patients; this value determines the maximum value the length of the queue to enter the facility can take before a newly arrived patient becomes completely discouraged and goes home before entering the queue in the first place. The line length tolerance was set to be normally distributed with a mean of twice the patience level and a standard deviation of 20 minutes. An hour before the facility closes at 6:00 p.m., the MIC stops taking new patients and closes its doors. Patients who were still outside at this time go home while the patients inside proceed through the MIC. The simulation tracks the number and percentage of patients who arrive seeking vaccination but who ultimately go home because of excessive wait times, discouraging line lengths, or arriving too late in the day. The simulation also tracks the earliest arrival time for which a patient failed to gain entry into the MIC because of facility closure to measure the latest a patient can arrive and still be treated.

\subsection{Modeling Risk of Disease Transmission}

Airborne influenza transmission is believed to occur only within a two-meter radius of the infected person (Brankston et al. 2007). Using transmission probability hazard rates for influenza estimated by Haber et al. (2007) (Table 3), the probability that a contagious patient will infect a susceptible patient or a non-infected-symptomatic patient by time $t$ can be estimated using the cumulative density function of an exponential distribution: $\operatorname{Pr}($ Infection $)=1-e^{\lambda_{i j} t}$, where $t$ is the amount of time the two patients spend within a two-meter radius of each other, and $\lambda_{i j}$ is the hazard rate for a minute of exposure that depends on the age groups $i$ and $j$ of the susceptible and infectious persons, respectively.

Table 3: Pandemic influenza transmission hazard rates $(\lambda)$ per minute (Haber et al., 2007).

\begin{tabular}{l|llll}
\hline & \multicolumn{4}{|c}{ Age Group of Susceptible Person } \\
\hline Age Group of Infectious Person & $0-4$ & $5-18$ & $19-64$ & $\geq 64$ \\
\hline $0-4$ & 0.00059 & 0.00062 & 0.00033 & 0.0008 \\
$5-18$ & 0.00058 & 0.00061 & 0.00033 & 0.0008 \\
$19-64$ & 0.00057 & 0.00053 & 0.00032 & 0.0008 \\
$\geq 64$ & 0.00057 & 0.00054 & 0.00029 & 0.00102 \\
\hline
\end{tabular}

Susceptible patients can be exposed to multiple infectious persons at a time. If it is assumed that the probability of being infected in an equal period of time of simultaneous contact with any $N$ infectious individuals is independent, then the overall hazard rate for that period can be represented as the sum of the $N$ hazard rates. Since the number and type of infectious person a susceptible person may be exposed to will change as s/he progresses through the simulation's work stations, it is necessary to re-assess with whom the susceptible person has contact every time his/her position in the simulation changes.

Previously, the probability of infection was completely determined by $\lambda_{i j} t$, a quantity that measures the susceptible patient's exposure to an infectious patient. A more granular representation of exposure is needed to reflect the different persons, hazard rates, and time intervals that may contribute to this quantity. Letting the set $J$ represent all infectious patients with whom a susceptible person $i$ has contact, and $K$ represent all time intervals over which the susceptible person occupies a distinct position in the MIC system, $\sum_{J, K} \lambda_{i j} t_{k}$, where $t_{k}$ represents the $k$ th time interval, can be used to represent the susceptible person's exposure. The probability of a susceptible person becoming infected in the MIC can therefore be represented as: $\operatorname{Pr}($ Infection $)=1-e^{\sum_{J, K} \lambda_{i j} t_{k}}$.

To track total contagious person exposure time, each susceptible person is assigned an exposure variable that gets updated at every time-step $k$. To update the exposure variable, the simulation must determine 


\section{Beeler, Aleman, and Carter}

which individuals are within two meters of a given susceptible individual, and then check their health status. A heuristic was used because recording the precise coordinates of each patient within an MIC floor plan could involve excessive and unnecessary computation while also requiring capabilities beyond what many process-oriented discrete event simulation software packages offer. To approximate the two-meter radius rule, the simulation checks the health status of individuals within $M$ ranks on either side of the individual's position in a queue during time $t_{k}$. In a baseline scenario, it is assumed that $M=3$ for queues inside the MIC and $M=4$ for queues outside the MIC entrance. The maximum number of people one can have contact with at a given time is therefore $2 M$. To represent greater congestion or adjacent single-file queues, $M$ can be increased. The simulation does not presently consider the probability of transmission to healthcare workers on duty since the transmission risk to individuals wearing protective masks is likely low. The cumulative number of infections per simulated day is computed as the sum of the probability of infection for each susceptible individual who passed through the system that day.

In very long queues, the people to whom a susceptible person is exposed is re-assessed every time the person's rank in the queue changes, even though this set of people may remain constant for up to 30-60 minutes. This superfluous calculation can be rectified by tracking the time a person spends three ranks away from the start and end of a line and multiplying that time by $\sum_{J} \lambda_{i j}$ rather than re-identifying each person within $M$ ranks and re-determining the relevant hazard rates. However, even without this reduction in computations, the model completes trials with a run time under 60 seconds.

\section{RESULTS}

\subsection{Baseline Scenario}

In the baseline scenario, staff allocations to the registration desks, vaccination stations, and flu assessment area were four, 10, and one, respectively. The percentage of infectious individuals in the system was set to $4 \%$, split equally between symptomatic and asymptomatic cases. The queue capacities in front of the registration desks, vaccination stations, and flu assessment areas were 20, 90, and 20, respectively. No limit was set on the number of patients who could wait outside, though patients could leave after waiting beyond their patience level to gain entry to the facility, or if the line length was beyond their line tolerance.

A trial with 10 replications was conducted to estimate the expected number of infections occurring per day, the total number of arrivals, and the total number of vaccines administered. Table 4 provides the mean and $95 \%$ confidence interval for the these parameters.

Table 4: Results from 10 replications of baseline scenario.

\begin{tabular}{l|ll}
\hline Parameter & Mean & 95\% Confidence interval \\
\hline E(Number of infections) & 9.14 & $(7.91,10.37)$ \\
Total arrivals & 1383.9 & $(1348.5,1419.3)$ \\
Vaccinations & 1146.1 & $(1141.1,1151.0)$ \\
\hline
\end{tabular}

The trials show that the expected number of infections occurring in the MIC is less than $1 \%$ of the number of people vaccinated, or about nine infections per day in a facility that vaccinates 1,000 patients daily. This count is high enough to merit giving attention to controlling transmission risk in MICs, but does not alter or meaningfully detract from the overall benefits of mass immunization. The variance in the number of people vaccinated per day is low because in all runs the MIC was working at full capacity. Staffing was not sufficient to handle all of the arrivals, leaving approximately 200 potential patients, or about $17 \%$ of arrivals, unable to gain admission before closing time or unable to tolerate the wait time or outdoor line length. 


\subsection{Designed Experiment}

The first 10 trials revealed that the standard deviation of the three response variables of interest was not wide, from a clinical or economic point of view. Indeed, 10 trials proved sufficient to obtain reasonably narrow confidence intervals for the expected number of infections, total arrivals and vaccinations. A three-factor full factorial design was used to test the effect of (1) increasing crowdedness, (2) staffing, and (3) the percentage of infectious patients in the system on the expected number of infections and the number of vaccinations issued. Crowdedness was represented by doubling the maximum rank difference to $M=6$ inside and $M=8$ outside, which could represent the switch from single-file lines to people forming lines in pairs, or simply twice the person-density per unit of floor area. Staffing was increased to five registration workers and 11 vaccination station workers, up from four and 10. The percentage of infectious symptomatic and asymptomatic patients entering the system was increased from $2 \%$ for each category to $4 \%$, holding constant the number of symptomatic non-infectious patients at $2 \%$.

In addition to the 10 baseline runs summarized previously, five additional runs were conducted for each of the seven remaining possible combinations of factor levels. Due to the low standard deviation of the response variables, additional runs were deemed unnecessary to detect clinically and economically significant statistical differences between the scenarios. For the impact on expected infections, the results from a two-way ANOVA showed no significant interactions between the factors, so a main effects model was fitted. A two-way ANOVA on the number of vaccinations completed per day revealed moderate evidence of an interaction between the staff increment and increased infectious patient prevalence. A linear model with an interaction between just these two factors was fit to obtain more precise estimates of the statistically significant coefficients. Table 5 summarizes the impact of the factors on expected infections, while Table 6 summarizes their impact on total vaccinations performed.

Table 5: Impact on expected infections.

\begin{tabular}{l|lll}
\hline Coefficients & Mean & $95 \%$ CI & p-value \\
\hline Intercept (baseline) & 9.98 & $(7.68,12.23)$ & $<0.001$ \\
Crowdedness & 11.06 & $(8.36,13.76)$ & $<0.001$ \\
More staff & -6.19 & $(-3.49,-8.89)$ & $<0.001$ \\
High infection rate & 10.65 & $(7.95,13.35)$ & $<0.001$ \\
\hline
\end{tabular}

The results in Table 5 indicate that the expected number of infections is twice the baseline level (9.98) when the proportion of infectious individuals doubles (adding 10.65), and when the number of persons with whom an individual has contact doubles (adding 11.06). Having one more staff person at the registration desk and vaccination stations reduced the expected number of infections by -6.19 -over a $60 \%$ decrease from the baseline scenario-by increasing the rate of patient throughput, and thereby reducing the average time spent waiting in a queue. All of these effects were highly statistically significant, with p-values under 0.001 .

Table 6: Impact on number of vaccines administered.

\begin{tabular}{l|lll}
\hline Coefficients & Mean & $95 \%$ CI & p-value \\
\hline Intercept (baseline) & 1146.9 & $(1134.6,1159.3)$ & $<0.001$ \\
Crowdedness & -8.55 & $(-22.1,5.0)$ & 0.192 \\
More staff & 104.9 & $(86.6,123.2)$ & $<0.001$ \\
High infection rate & -24.8 & $(-43.12,-6.5)$ & 0.00697 \\
Interaction: Staff $\times$ High inf. rate & -35.9 & $(-62.9,-8.8)$ & 0.00810 \\
\hline
\end{tabular}

According to the results in Table 6, crowding did not have a statistically significant effect on vaccine administration $(\mathrm{p}=0.192>0.05)$. Adding two extra staff significantly increased the average number of vaccines administered by 104.9 from the baseline of $1,146.9$, but the impact was not as strong when the 
infection rate was elevated $(104.9-35.9=69)$. This is likely because the gains in vaccinations from adding staff could not be fully realized when a larger proportion of patients used up registration time only to be sent to the flu-assessment area.

\section{CONCLUSIONS}

This simulation study is the first of its kind to attempt to estimate the risk of influenza transmission to healthy patients in a mass immunization clinic. The simulation's output indicate that the number of infections expected in an MIC will likely be under $1 \%$ of the number of people vaccinated during a pandemic of medium severity, but may rise above $2 \%$ under circumstances of crowding, under-staffing leading to long waits, and high infection prevalence in the general population.

For a large facility vaccinating over 1,000 patients per day, this could amount to over 20 infections and may warrant measures to reduce transmission risk, such as separating adjacent queues to be over 2 meters apart, ensuring MIC facility floor space is adequate to avoid crowding, ensuring adequate staffing to control wait times, effectively discouraging patients with flu-like symptoms from coming to MICs or identifying them before they spend significant time waiting in queues, and sending them home or to flu assessment centers if they do arrive.

As with all simulation studies, the results presented are contingent on the model's parameters and numerous simplifying assumptions. Significant potential exists for gains in model accuracy and precision on several fronts. Transmission hazard rates within a two-meter radius are likely not well represented by an age-dependent set of constants, as they were in this study, but are likely an inverse quadratic or inverse cubic function of the distance between the infectious and susceptible persons. This is because virus-laden droplet density in the air will become more diffuse as the planar surface area, or volume, of space surrounding an infectious person increases with distance. The differences between transmission risk from symptomatic vs. asymptomatic patients was not considered, nor was the difference in risk borne by persons in front of vs. behind an infectious person. Many of these epidemiological parameters are currently unknown or estimated only with high degrees of uncertainty. The parameters in the model presented can be updated easily to reflect advances in epidemiology. The provisional infection risk estimates produced by this simulation model are nonetheless useful for comparing MIC scenarios, and for identifying roughly where the true values for MIC infection outcomes could lie.

\section{REFERENCES}

Aleman, D., T. Wibisono, and B. Schwartz. 2010. "A non-homogeneous mixing model for predicting pandemic spread". Interfaces Special Issue on Humanitarian Applications: Doing Good with OR 41:301315.

Araz, O., T. Lant, J. Fowler, and M. Jehn. 2011. "A simulation model for policy decision analysis: a case of pandemic influenza on a university campus". Journal of Simulation 5 (2): 89-100.

Barnes, S., B. Golden, and E. Wasil. 2010, December. "A dynamic patient network model of hospitalacquired infections". In Proceedings of the 2010 Winter Simulation Conference, edited by B. Johansson, S. Jain, J. Montoya-Torres, J. Hugan, and E. Yücesan, 2249-60. Piscataway, New Jersey: Institute of Electrical and Electronics Engineers, Inc.

Brankston, G., L. Gitterman, Z. Hirji, C. Lemieux, and M. Gardam. 2007. "Transmission of influenza A in human beings". Lancet Infectious Disease 7:257-265.

H. Branswell 2009, December. "Swine flu in 1976 led to Canada's pandemic vaccine contract". http://www.healthzone.ca/health/newsfeatures/article/ 667963--swine-flu-in-1976-led-to-canada-s-pandemic-vaccine-contract [accessed March 15, 2011 ].

Statistics Canada 2006. "Census 2006”. http://www12.statcan.ca/census-recensement/2006/rt-td/index-eng. cfm [accessed March 15, 2011]. 
Das, T., A. Savachkin, and Y. Zhu. 2008. "A large scale simulation model of pandemic influenza outbreaks for development of dynamic mitigation strategies". IIE Transactions 40 (9): 893-905.

Haber, M., D. Shay, X. Davis, R. Patel, X. Jin, E. Wientraub, E. Orenstein, and W. Thompson. 2007. "Effectiveness of interventions to reduce contact rates during a simulated influenza pandemic". Emerging Infectious Disease 13:581-589.

Khan, K., J. Arino, F. Calderon, A. Chan, M. Gardam, C. Heidebrecht, W. Hu, D. Janes, M. MacDonald, and J. Sears. 2011. "An Analysis of Canada's Vulnerability to Emerging Infectious Disease Threats via the Global Airline Transportation Network". Technical report, St. Michael's Hospital. http://www2. biodiaspora.com/low_res.pdf [accessed April 1, 2011].

Mei, S., D. Van De Vijver, L. Xuan, Y. Zhu, and P. Sloot. 2010, June. "Quantitatively evaluating interventions in the influenza A (H1N1) epidemic on China campus grounded on individual-based simulations". In Proceedings of the 10th International Conference on Computational Science, edited by P. Sloot, D. van Albada, and J. Dongarra, 1675-1682. International Conference on Computational Science 2010.

Noakes, C., and P. Sleigh. 2009. "Mathematical models for assessing the role of airflow on the risk of airborne infection in hospital wards". Journal of the Royal Society, Interface 6:S791-S800.

Stroud, P., S. Del Valle, S. Sydoriak, J. Riese, and S. Mniszewski. 2007. "Spatial dynamics of pandemic influenza in a massive artificial society". Journal of Artificial Societies and Social Simulation 10 (4): 9.

Stroud, P. D. 2006. "EpiSimS Los Angeles Case Study". Technical report, Los Alamos National Laboratory. http://public.lanl.gov/stroud/LACaseStudy5.pdf [accessed March 15, 2011].

\section{AUTHOR BIOGRAPHIES}

MICHAEL F. BEELER is a graduate student in the Department of Mechanical and Industrial Engineering at the University of Toronto. He is also a CIHR-STIHR Public Health Policy Fellow and Executive Director of Students for International Development. His research interests are pandemic planning and non-profit supply chain management. Michael is a member of INFORMS, INFORMS Simulation Society and the Systems Dynamics Society. His e-mail is michael.beeler@utoronto.ca.

DIONNE M. ALEMAN is an Assistant Professor in the Department of Mechanical and Industrial Engineering at the University of Toronto. Her research interests are medical applications of operations research, specifically, pandemic planning and radiotherapy treatment optimization. She serves as the President of the INFORMS Junior Faculty Interest Group and the Secretary and Treasurer of the INFORMS Section on Public Programs, Services, and Needs, and is a past Chair of the INFORMS Health Applications Section. Prof. Aleman is an Associate Editor for IIE Transactions on Healthcare Systems Engineering and the Journal of Biomedical Data Mining. She is also the Director of the Medical Operations research Lab (morLAB) at the University of Toronto. Professor Aleman's email address is aleman@mie.utoronto.ca.

MICHAEL W. CARTER is a Professor in the Department of Mechanical and Industrial Engineering at the University of Toronto, and Director of the Centre for Research in Health Care Engineering. His research interests are healthcare resource modeling, demand forecasting and scheduling. He is on the editorial board for the Journal of Scheduling and the journal Health Care Management Science, and is an Adjunct Scientist with the Institute for Clinical Evaluative Sciences in Toronto (www.ices.on.ca). Professor Carter's email address is carter@mie.utoronto.ca. 